\title{
Facial emotion recognition using deep convolutional neural network and smoothing, mixture filters applied during preprocessing stage
}

\author{
Pragnyaban Mishra, P. V. V. S. Srinivas
}

Department of CSE, Koneru Lakshmaiah Education Foundation, Vaddeswaram, Guntur District, Andhra Pradesh, India

\begin{tabular}{l}
\hline \hline Article Info \\
\hline Article history: \\
Received Oct 20, 2020 \\
Revised Sep 18, 2021 \\
Accepted Oct 1, 2021 \\
\hline
\end{tabular}

Keywords:

Classification

Deep convolutional neural network

Facial emotion recognition Image smoothing

\begin{abstract}
The facial emotion recognition by the machine is a challenging task. From decades, researchers applied different methods to classify facial emotion into the different classes. The expansion of artificial intelligence in a form of deep convolutional neural network $(\mathrm{CNN})$ changed the direction of the research. The facial emotion recognition using deep $\mathrm{CNN}$ is powerful in terms of taking bulk input images for processing and classify with high accuracy. It has been noticed in a few cases the classification model does not judge the facial images into appropriate classes due to the influence of noises. So, it is highly recommended to apply a noiseless image to the facial emotion recognition model for classification. We adopted a mechanism and proposed a model for classifying facial image into one of the seven classes with high accuracy. The images are smoothed before applying to the model by different smoothing process as part of image preprocessing. We claim facial emotion recognition with image smoothing by different filters or a mixture of filter are more robust than without preprocessing. The detail is explained in the subsequent sections.
\end{abstract}

This is an open access article under the CC BY-SA license.

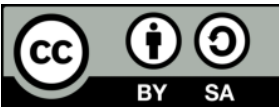

\section{Corresponding Author:}

Pragnyaban Mishra

Department of CSE

Koneru Lakshmaiah Education Foundation

Vaddeswaram, Guntur District, Andhra Pradesh, India, 522501

Email: pragnyaban@kluniversity.in

\section{INTRODUCTION}

Image is a set of pixels, represented by the function $f(x, y)$ such that, $x \in \operatorname{domain}(x-\operatorname{axis})$ and $y \in$ range $(y-a x i s)$ of an image having the scalar quantity, is equivalent to the amount of energy radiated from the place image is taken. Suppose $f(a, b)$ designates an image of continuous variables which is converted into digital image in a form of $f(x, y)$ where $x \in\{0,1,2, \ldots, \mathrm{M}-1\}$ and $y \in\{0,1,2, \ldots, \mathrm{N}-1\}$ Here $\mathrm{M}, \mathrm{N}$ are the length and breadth of the digital image. Following is the matrix representation of above image definition in (1):

$$
f(x, y)=\left(\begin{array}{ccc}
f(0,0) & \cdots & f(0, M-1) \\
\vdots & \ddots & \vdots \\
f(N-1,0) & \cdots & f(N-1, M-1)
\end{array}\right)
$$

Here, each of $f\left(x_{i}, y_{i}\right)$ represents spatially to a pixel of $\left(x_{i}, y_{i}\right)$. For every pixel $\left(x_{i}, y_{j}\right)$ such that $0 \leq i, j \leq M, N$. The four neighboring pixels of any of the pixels $\left(x_{i}, y_{j}\right)$ are represented by 
$\left(x_{i+1}, y_{j}\right),\left(x_{i-1}, y_{j}\right),\left(x_{i}, y_{j+1}\right),\left(x_{i}, y_{j-1}\right)$. Often, the image cannot be analyzed in true sense due to its bad quality and amount of the noise present [1]. The corrupted image is presented as (2):

$$
g(x, y)=f(x, y)+\eta(x, y)
$$

where $f(x, y)$ : Noiseless image and $\eta(x, y)$ : Noises present in the image.

The presence of noise corrupts partially or in a regularly at different portions of the image. As a result, the image knowledge extraction may not be in a true sense. For recovery, the quality of the image from the noise image filtering is used. According to [2] there are several filters like average, median, gaussian, and bilateral are used to smooth the image. In this situation the convolution is used and is represented by operator $\circledast$ applied on $f(x, y)$ with the impulse response of $g(x, y)$ create smooth image $h(x, y)$ explained as $(3)$.

$$
\begin{aligned}
& h(x, y)=f(x, y) \circledast g(x, y)=\int_{-\infty}^{+\infty} \int_{-\infty}^{+\infty} f\left(x^{\prime}, y^{\prime}\right) g\left(x-x^{\prime}, y-y^{\prime}\right) d x^{\prime} d y^{\prime} \\
& h[i, j]=f[i, j] \circledast g[i, j]
\end{aligned}
$$

The human face represents some sensible information which changes from time to time [3] with external or internal influence. In this article we have demonstrated the facial emotion recognition model by applying artificial intelligence. The input to this model is filtered by different filters as a part of image preprocessing that lead by higher accuracy compared without smoothing. The facial emotion recognition begins from Darwin, [4] said there are 40 human expressions curves a human face poses after perceiving inputs from the environment. The action units [5], [6] of the face are the fundamental unit of the expression which contain sensitive information of expression. Convolutional neural network (CNN) consist of convolutional layer, pooling layer and fully connected network [7] is the most interesting tool and technology that, produces promising result [8] for any high-level scientific computation [9], [10]. Convolutional neural networks are not only for facial emotion recognition that we applied in the under described research, but also in several classifications such as human disease classification [11], [12], and plant disease classification [13]. Before deep CNN quite popular, the image classification uses a different machine learning algorithms and methods to classify in applications like brain tumor [14], [15], Plant disease [16], [17] and other [18], [19].

We have adopted a deep CNN in our research. The input to the architecture is preprocessed facial image which is filtered by various filters [20] as a result the quality of the image is enhanced. Filters have different measures for smoothing the image by removing impulse noise as per the function it uses. The convolutional neural network accepts smoothed image and train an artificial intelligence model for facial emotion recognition that is either happy, sad, fear, disgust, neutral, surprise and angry. In the general complexity of the model increases and accuracy decreases as number of the classes increases that are more challenging. We claim our model stood well for a wide variety of emotion classification with high accuracy.

The primary input to facial emotion recognition model is an image. The training of the model is influenced depending on the amount of noises are in the images. It is believed that the smoothed image is more robust than not. The filters that smooth images are average, median, gaussian and bilateral each filter have its own pros and cons. However, most of them cannot well recover a heavy noise corrupted image with noise density above $70 \%$ to preserve the detailed information of an image [21]. The median filter and its different variants are extensively used [22] to reduce the impulse noise from grayscale images and the performance is increased. Averaging the pixel intensities with respect to the size of the filter is a common method for smoothing the image, but fuzzy averaging [23] reduces impulses in a large way. Identify the pixels belonging to the borders, then apply a reduced smoothing and applying more intense smoothing to the remaining pixels produced a standard result [24] in the ultrasound image application.

The median filtering is a good choice of noise reduction. An improved median filtering algorithm [25] uses the correlation of the image to process the features of the filtering mask over the image. Median filtering based on combined features of different image that, consist of joint conditional probability density functions, principal component analysis is used to reduce the dimension is performing on the uncompressed image datasets. A new proposed method [26] uses a median filter using prior information to capture natural pixels for restoration, this method restores corrupted images with $99 \%$ level of salt-and-pepper impulse noise. Switching among the median and mean [27] by detecting a filter is a proved method of smoothing.

Gaussian function used for gaussian blur [28], is a kind of normal distribution. The original pixel having the highest intensity is replaced by maximum gaussian weight and proportionally the lower intensity

Int J Artif Intell, Vol. 10, No. 4, December 2021: 889 - 900 
is replaced by low gaussian weight. The review article [29] is a good collection of gaussian filers used in different applications and explained the advantages of this filter with respect to others.

The noise reduction along with preserving edge information [30] smoothing achieves using the [31] bilateral filter. Here the intensity of each of the pixel is substituted by a weighted average of an intensity calculated from the nearby pixels. The framework for image denoising [32] and suppresses mixed noise in color images [33] are a few of the advance example using the bilateral filter. The remaining of the paper is organized into the sections as: Section 2: Research method, section 3: Result and discussion and section 4: Conclusion.

\section{RESEARCH METHOD}

\subsection{Dataset description}

The renowned datasets FER2013 and CK48+ datasets are used for experimentation in the proposed model. CK48+, Fer2013 datasets consists of 3540, 35887 images related to seven different facial expressions such as happy, angry, sad, surprise, neutral, disgust, and fear, respectively. All the images are normalized, standardized by using standardization and normalization techniques, all the images are resized into a fixed dimension of $48 \mathrm{X} 48$ to maintain uniformity.

\subsection{Filter description}

The basic focus of our research is to observe facial emotion classification and its accuracy achievements for smoothed input images. The images undergone through different smoothing process and observation is tabulated in experimental section. For smoothing the images, a hybrid smoothing filter is proposed which is formed by the combination of average, median, gaussian, bilateral filters and their performances are compared. The equations used in each of the filters are as mentioned is: average filtering in (5), median filtering in (6), gaussian in (7) for 1D and in (8) for 2D, bilateral in (9),

$$
\begin{aligned}
& \operatorname{Img}(x, y)=\sum_{j=-1}^{1} \sum_{i=-1}^{1} 1 * \operatorname{Img}_{A c t}(x+i, y+j) \\
& \operatorname{Im} g_{n o r m(x, y)}=\frac{1}{\sum_{j=-1}^{1} \sum_{i=-1}^{1}} \operatorname{Img}(x, y) \\
& \operatorname{Img}(x, y)=\operatorname{median}\left\{\operatorname{Img} g_{a c t}((x+i, y+j) \mid(i, j \in R))\right\} \\
& G(x)=\frac{1}{\sqrt{2 \pi \sigma^{2}}} e^{\frac{-x^{2}}{2 \sigma^{2}}} \\
& G(x, y)=\frac{1}{2 \pi \sigma^{2}} e^{\frac{-\left(x^{2}+y^{2}\right)}{2 \sigma^{2}}} \\
& \operatorname{Img} \text { Filtered }=\frac{1}{W_{t}} \sum \operatorname{Img}\left(x_{i}\right) f_{\text {range }}\left(\bmod \left(\operatorname{Img}\left(x_{i}\right)-\operatorname{Img}(x)\right) G_{x}\left(\bmod \left(x_{i}-x\right)\right)\right. \\
& W_{t}=\sum f_{\text {range }}\left(\bmod \left(\operatorname{Img}\left(x_{i}\right)-\operatorname{Img}(x)\right) G_{x}\left(\bmod \left(x_{i}-x\right)\right)\right.
\end{aligned}
$$

\subsection{Model description}

In the devised model a facial emotion recognition image dataset is taken and is converted to a hybrid image set by applying various smoothing techniques.

Step 1: Initially, $\mathrm{n}$ random images from the image set is selected by using randSelect function proposed in the algorithm.

Step 2: Average filtering is applied on the randomly selected images and the resulted images are stored in hybrid image set, the random images selected are removed from the original image set.

Step 3: The same process is repeated by using median, gaussian, and bilateral filters and a hybrid image set is formed from different filtered images.

Step 4: Assign labels to the resulted hybrid image set

Step 5: Divide the hybrid image set in the ratio of 80:15 for training and testing purpose

Step 6: Train the proposed CNN model with selected images for training and evaluate with the images selected for testing for training and evaluation.

\subsubsection{Algorithm}

The stages in the algorithm illustrate the process in evaluating a face picture as an input into an emotion class. The algorithm uses three functions: hybrid filtering, randSelect, and FacEmoRec. The 
hybrid filtering function chooses pictures that are filtered using average, median, bilateral, and gaussian methods. FacEmoRec classifies photos based on emotion using the randSelect function, which randomly picks photos from the original dataset on which filtering should be done.

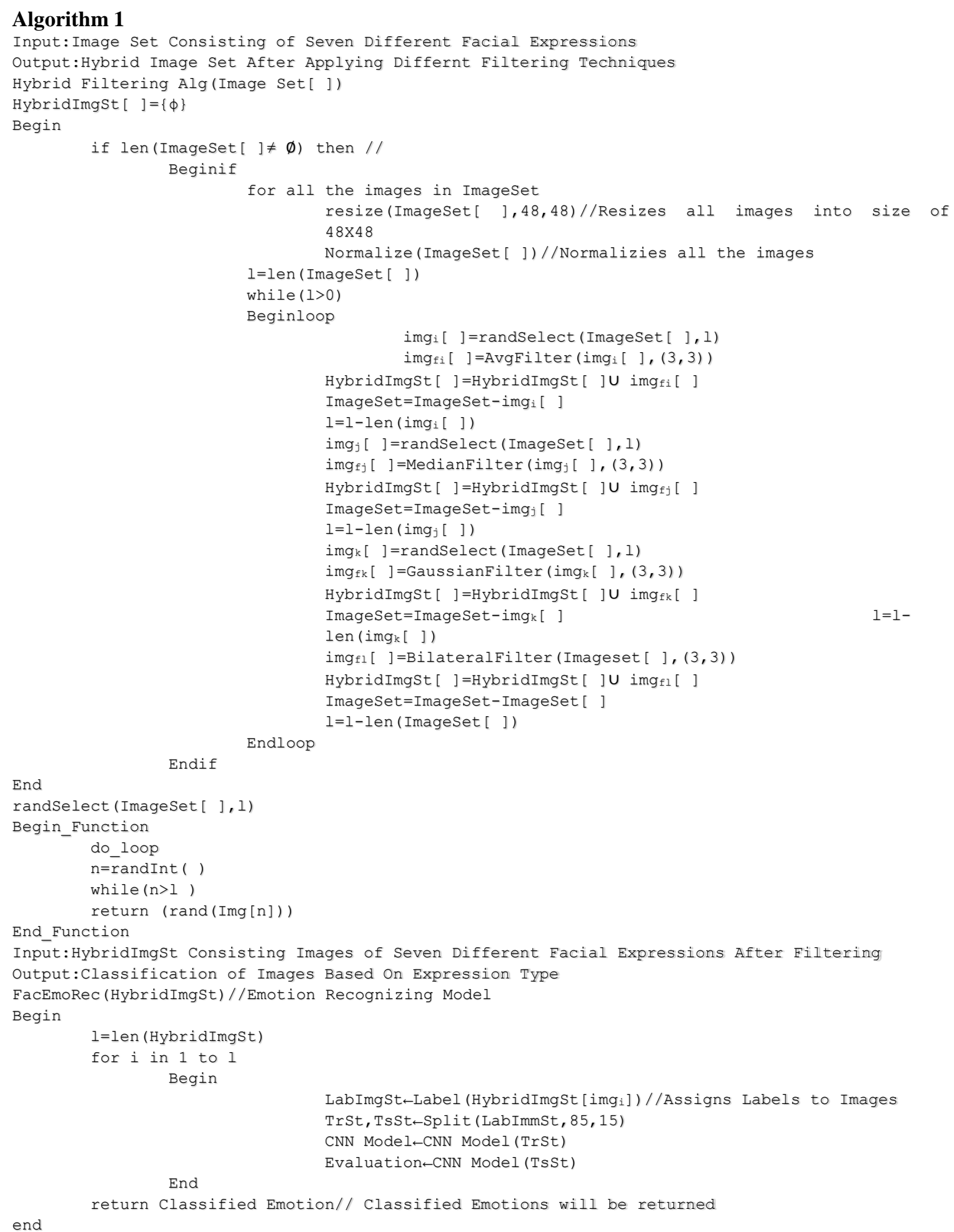

\subsubsection{Flow chart for the proposed model}

Figure 1 describes the application of different filters, average, median, gaussian, and bilateral to the image dataset consist of finite images. All images passed through different filters are equal to the total number of images in the actual dataset. The filtered images are applied to the model for training and evaluation. 


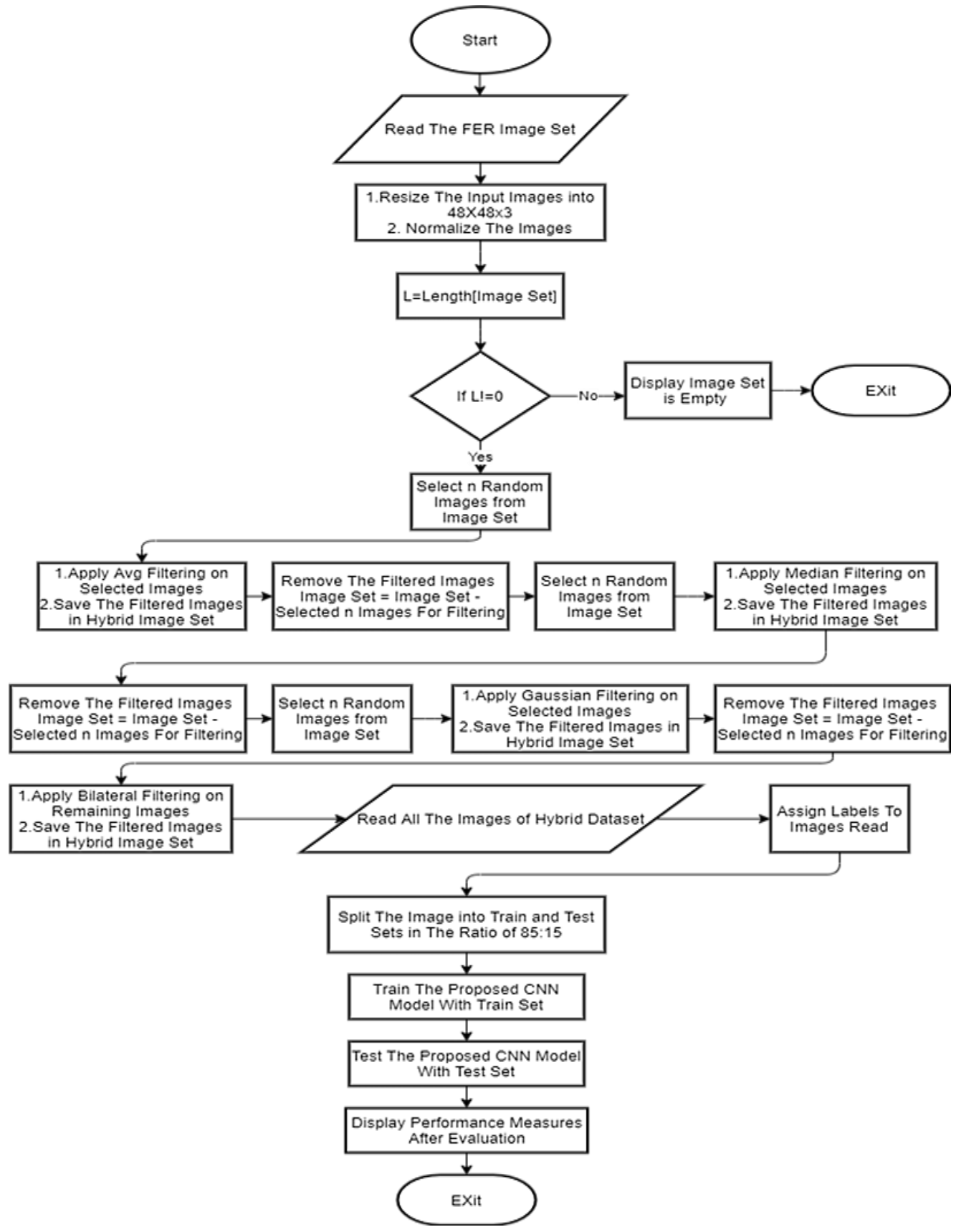

Figure 1. Develop a model with a hybrid dataset whose performance is measured through a proper evaluation plan

\section{RESULTS AND DISCUSSION}

High computation speeds in terms of graphical processing unit (GPU), central processing unit (CPU) and memory are required to build a hybrid image filter algorithm and to build a CNN model for evaluating the performances of the hybrid image filter dataset. We took the support of Google Colab cloud service support for developing the above-mentioned models. The configuration of the cloud service used is described as:

Frequency of CPU: $2.30 \mathrm{GHz}$, GPU Used: NIVIDIA (12GB), Size of Disk Space Supported: 25 GB, Editor Used: Jupiter Notebook. CK48+, Fer2013 datasets that consists of 3540, 35887 images related to seven different facial expressions such as happy, angry, sad, surprise, neutral, disgust, and fear are considered for experimentation. Average, median, gaussian, bilateral and the proposed filter hybrid filters are considered for filtering the datasets and the resulted images are given for a CNN model for evaluation. It is observed that the images that were considered as inputs to the CNN model after applying 
filtering produced better results when compared to the images where filtering is not applied produced better results when compared to the images where filtering is not applied. Figure 2 represents accuracy and loss comparisons that are obtained from the model without filtering and with average and median filtering techniques applied to CK48+ dataset. Figure 2(a) represents train and test loss comparisons without filtering, Figure 2(b) represents train and loss comparisons when Average filtering is applied and Figure 2(c) represents train and test loss applied when median filtering is applied on CK48+ dataset. Figure 3 represents accuracy and loss comparisons that are obtained from the model with gaussian, bilateral and proposed hybrid filtering techniques applied to CK48+ dataset. Figure 3(a) represents train and test loss comparisons of gaussian filtering, Figure 3(b) represents train and loss comparisons when bilateral filtering is applied and Figure 3(c) represents train and test loss applied when hybrid filtering is applied on CK48+ dataset.
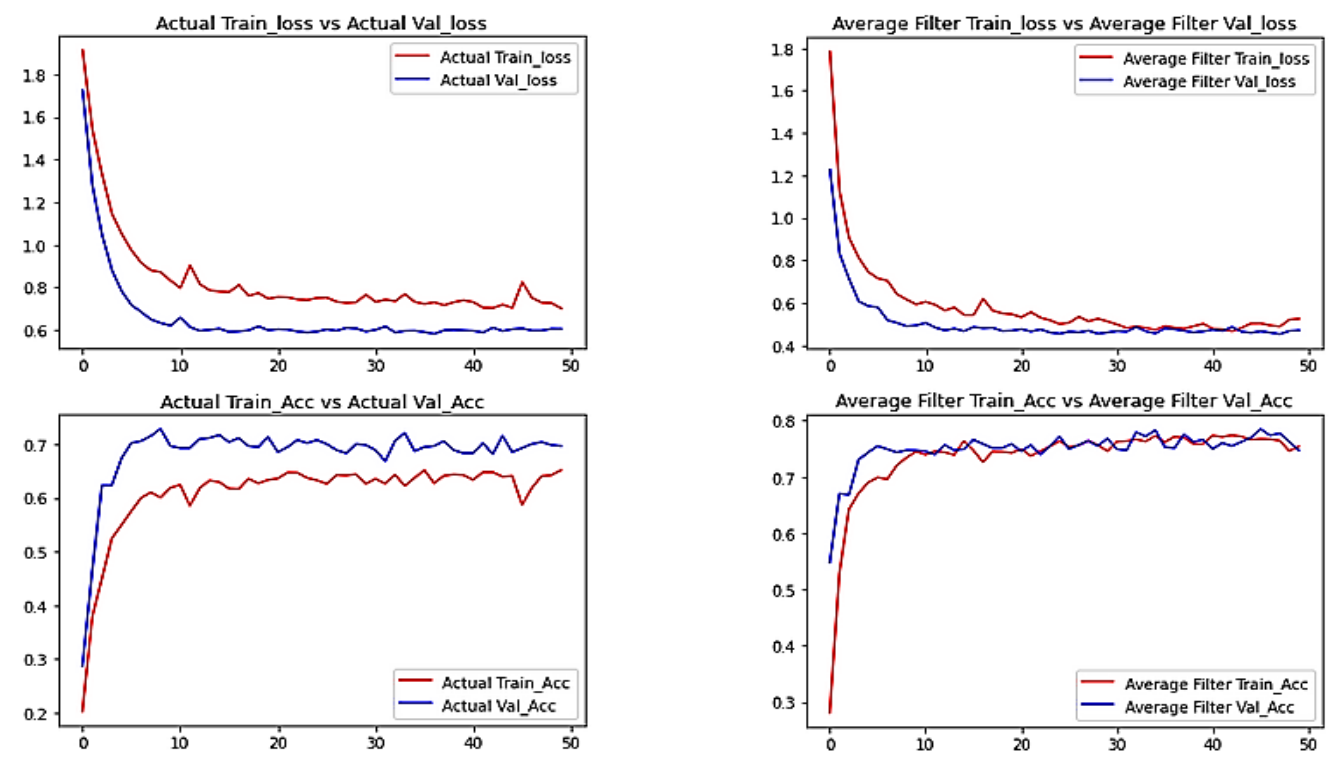

(a)

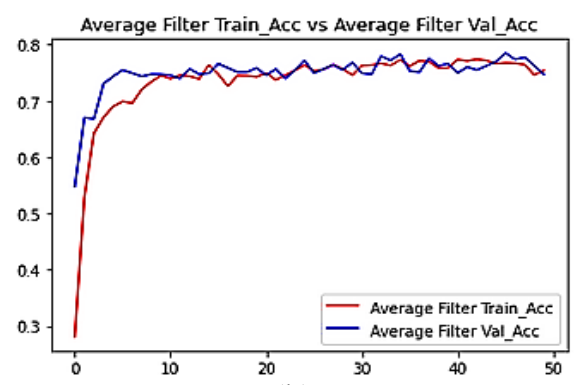

(b)
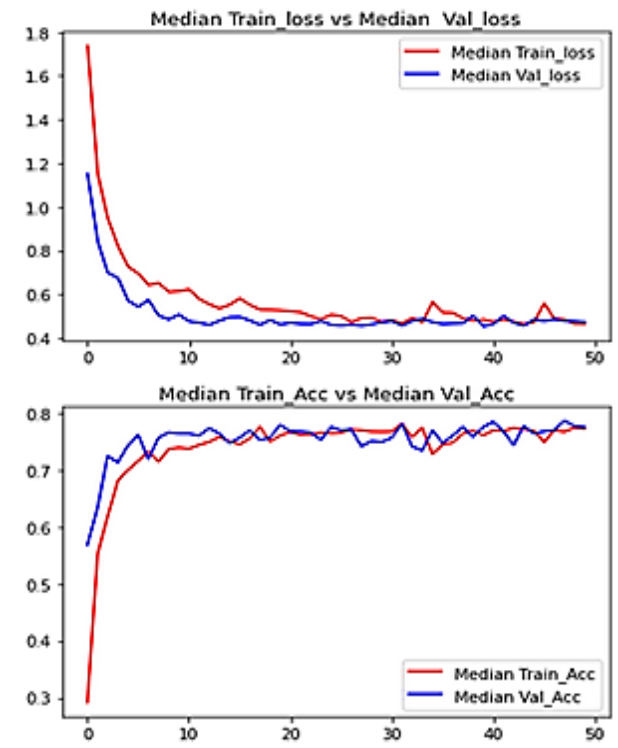

(c)

Figure 2. Performance measures without filtering and applying average and mean filtering on CK48+ dataset; (a) performance measures without applying filtering on CK48+ dataset, (b) performance measures after applying average filtering on CK48+ data set and (c) performance measures after applying median filtering on CK48+ dataset 

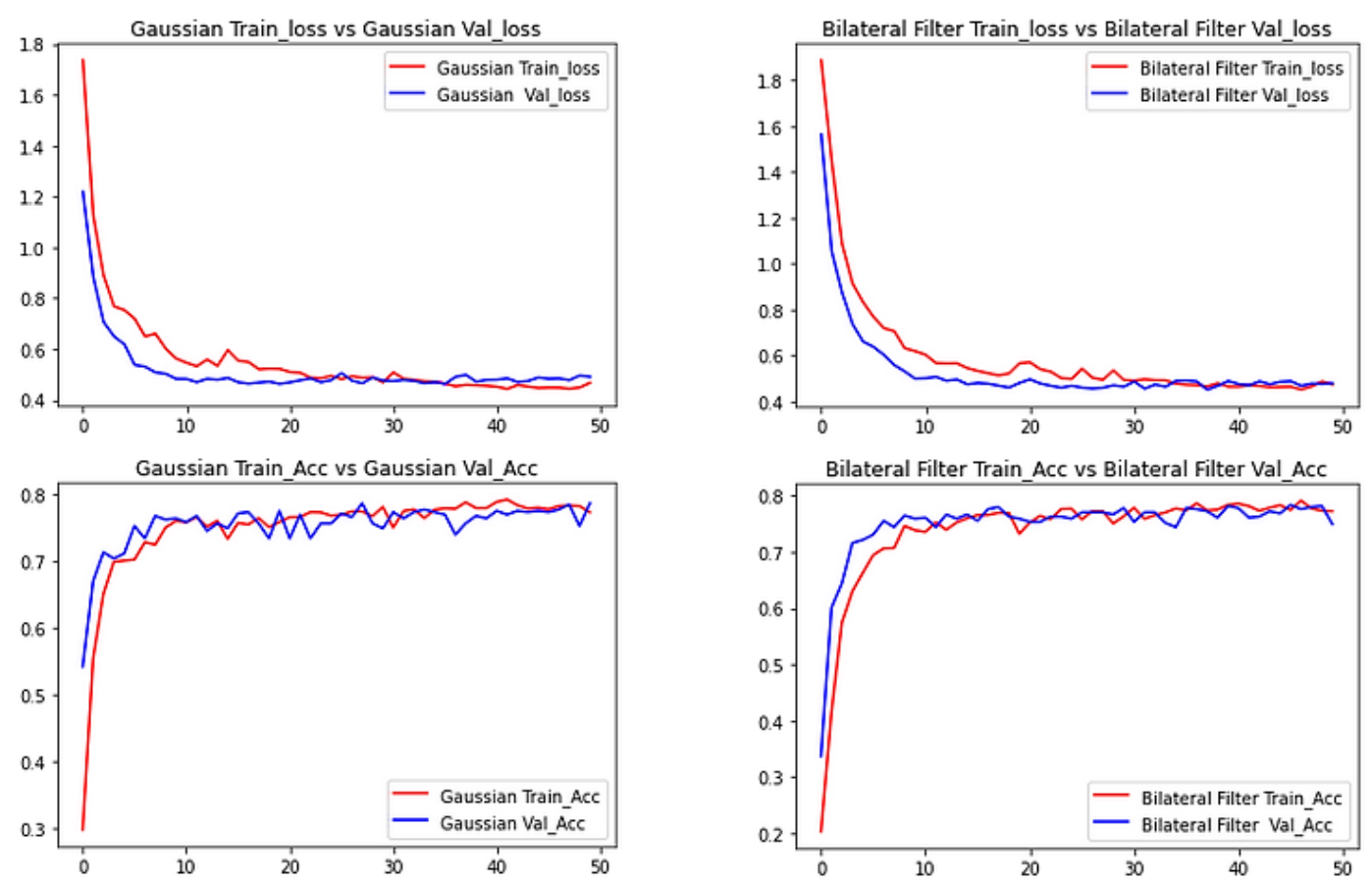

(a)

(b)
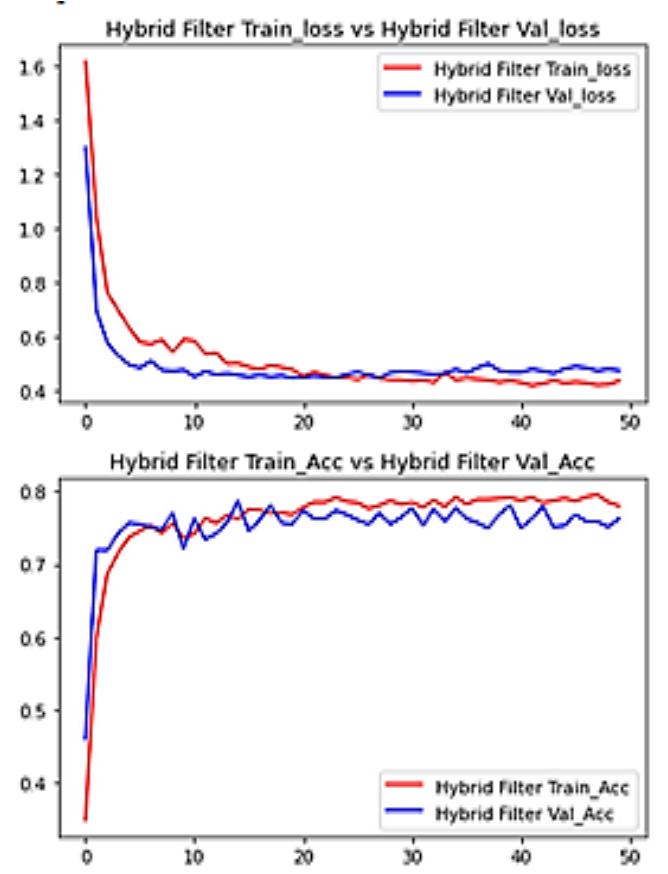

(c)

Figure 3. Performance measures after applying gaussian, bilateral and hybrid filtering on CK48+ dataset; (a) performance measures after applying gaussian filtering on CK48+ dataset, (b) performance measures after applying bilateral filtering on CK48+ dataset and (c) performance measures after applying hybrid filtering on CK48+ dataset

The Figure 4 represents accuracy and loss comparisons that are obtained from the model without filtering and with average and median filtering techniques applied to FER2013 dataset. Figure 4(a) represents train and test loss comparisons without filtering, Figure 4(b) represents train and loss comparisons when Average filtering is applied and Figure 4(c) represents train and test loss applied when median filtering is applied on FER2013 dataset. Figure 5 represents accuracy and loss comparisons that are obtained from the model with gaussian, bilateral and proposed hybrid filtering techniques applied to 
FER2013 dataset. Figure 5(a) represents train and test loss comparisons of gaussian filtering, Figure 5(b) represents train and loss comparisons when bilateral filtering is applied and Figure 5(c) represents train and test loss applied when hybrid filtering is applied on FER2013 dataset. Table 1 expresses the performance comparative analysis of train and test accuracy, loss and time taken for each epoch execution of the model with filtering and without filtering compared to the proposed hybrid filtering technique applied on FER2013 dataset.
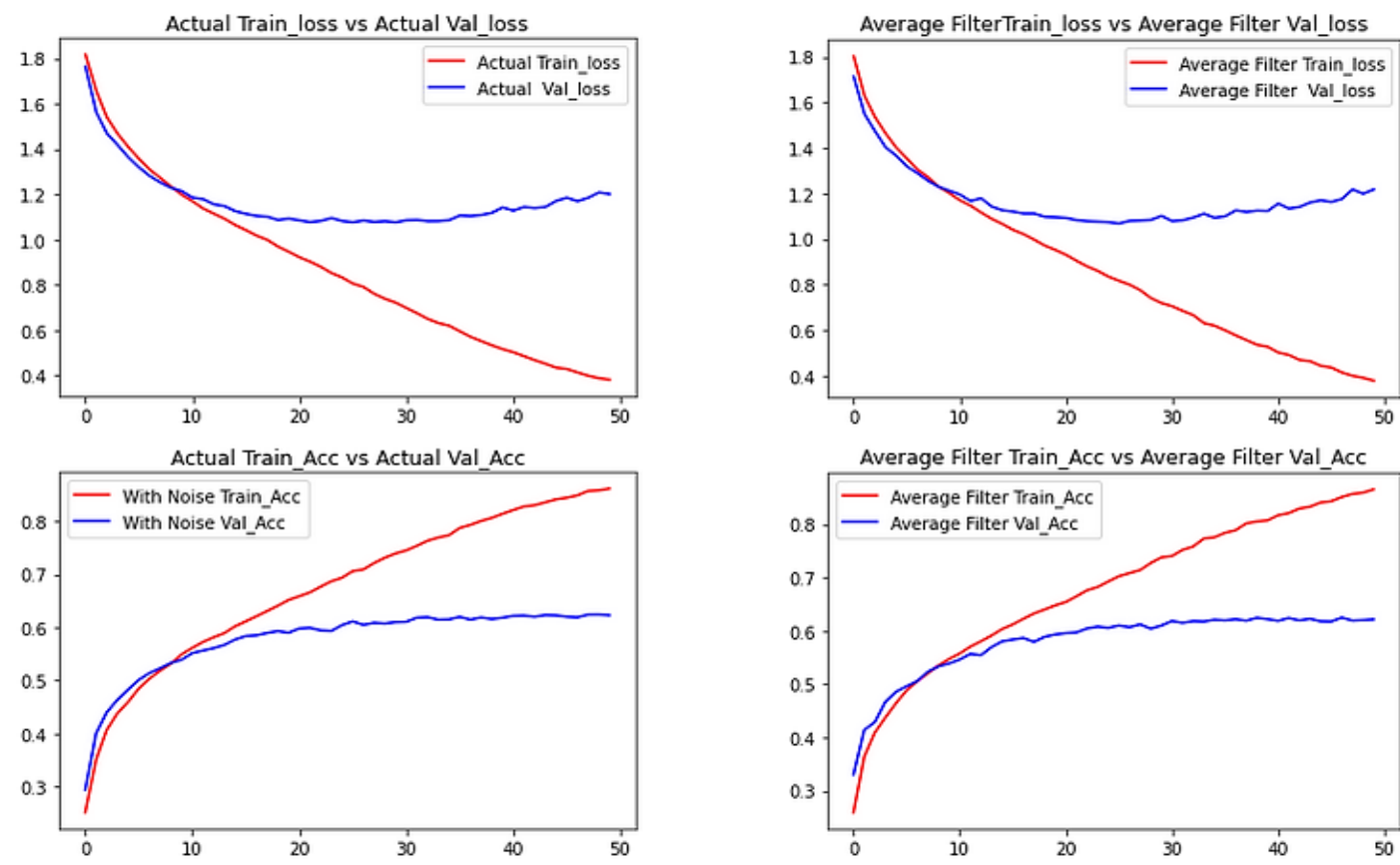

(a)

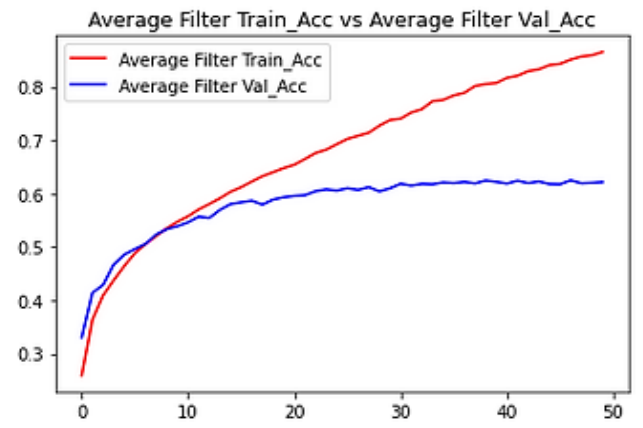

(b)
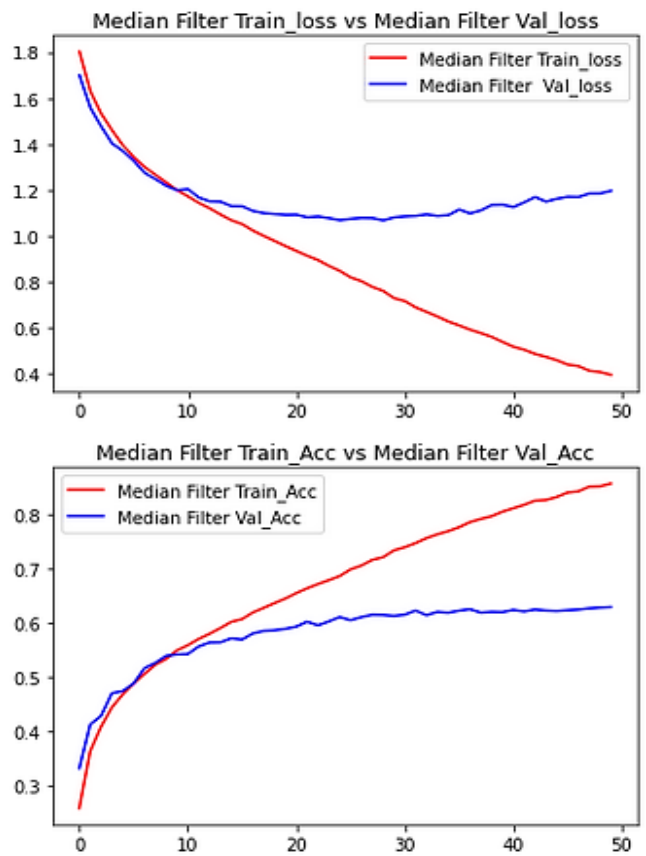

(c)

Figure 4. Performance measures without filtering and applying average and mean filtering on FER2013 dataset; (a) performance measures without applying filtering on FER2013 data set, (b) performance measures after applying average filtering on FER2013 data set and (c) performance measures after applying median filtering on FER2013 dataset 
Table 2 expresses the performance comparative analysis of train and test accuracy, loss and time taken for each epoch execution of the model with filtering and without filtering compared to the proposed hybrid filtering technique applied on CK48+ dataset. Figure 6 is a bar chart of accuracy levels that are obtained from the model with filtering and without filtering compared to the proposed Hybrid filtering technique applied on CK48+ and FER2013 datasets. Figure 7 is a bar chart of loss levels that are obtained from the model with filtering and without filtering compared to the proposed hybrid filtering technique applied on CK48+ and FER2013 datasets.
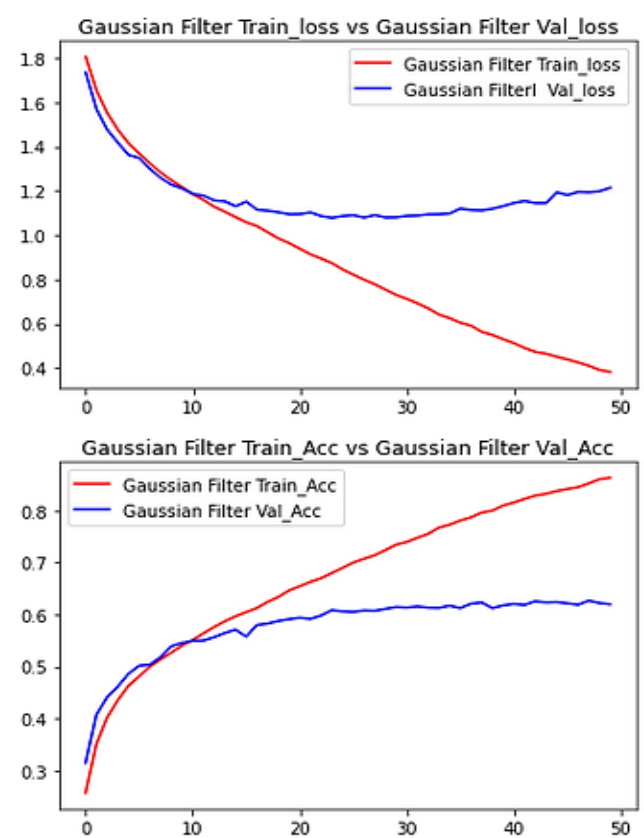

(a)

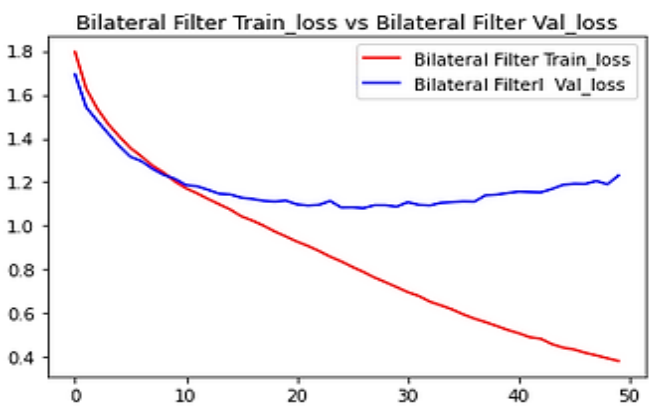

Bilateral Filter Train_Acc vs Bilateral Filter Val_Acc

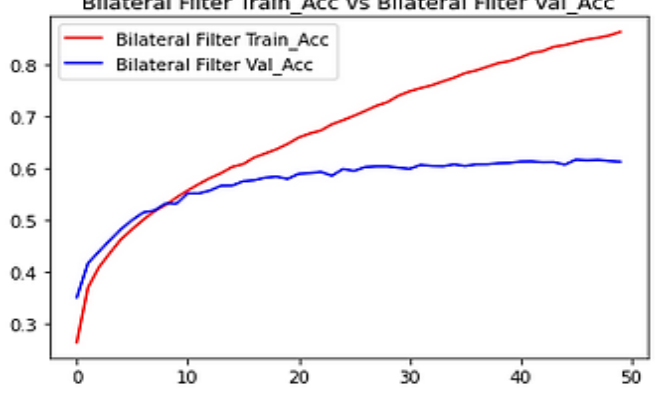

(b)

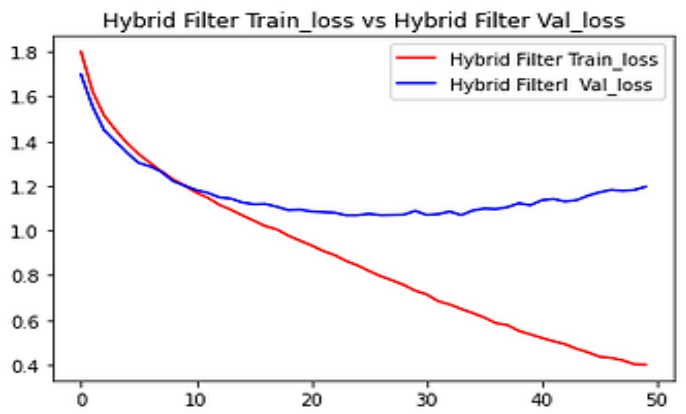

Hybrid Filter Train_Acc vs Hybrid Filter Val_Acc

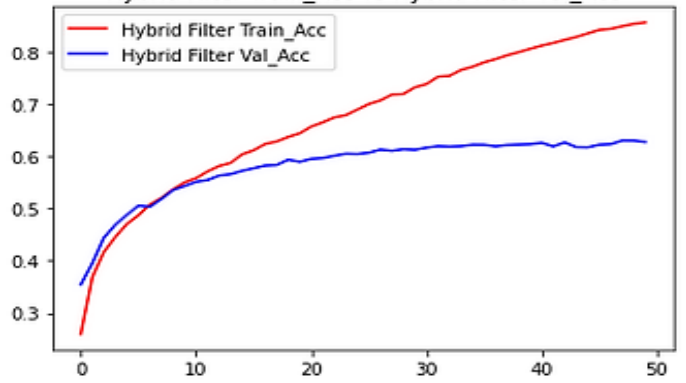

(c)

Figure 5. Performance measures after applying gaussian, bilateral and hybrid filtering on FER2013 dataset, (a) performance measures after applying gaussian filtering on FER2013 data set, (b) performance measures after applying bilateral filtering on FER2013 data set and (c) performance measures after applying hybrid filtering on FER2013 dataset 
Table 1. FER2013 performance comparative analysis using various filters

\begin{tabular}{ccccccc}
\hline S.No & Type of filtering applied & Train Accuracy & $\begin{array}{c}\text { FER-2013 } \\
\text { Test Accuracy }\end{array}$ & Train loss & Test loss & Exec time per epoch \\
\hline 1 & Average Filter & 86.2 & 61.89 & 0.383 & 1.201 & $17 \mathrm{Sec}$ \\
2 & Median Filter & 85.81 & 62.54 & 0.934 & 1.169 & $16 \mathrm{Sec}$ \\
3 & Gaussian Filter & 86.34 & 62.67 & 0.381 & 1.193 & $16 \mathrm{Sec}$ \\
4 & Bilateral Filter & 84.34 & 61.65 & 0.434 & 1.193 & $12 \mathrm{Sec}$ \\
5 & Hybrid Filter & 85.66 & 63.37 & 0.400 & 1.178 & $10 \mathrm{Sec}$ \\
6 & Without Filtering & 86.21 & 61.57 & 0.382 & 1.118 & $17 \mathrm{Sec}$ \\
\hline
\end{tabular}

Table 2. CK48+ performance comparative analysis using various filters

\begin{tabular}{ccccccc}
\hline & & \multicolumn{5}{c}{ CK $48+$} \\
S.No & Type of filtering applied & Train accuracy & Test accuracy & Train loss & Test loss & Exec time per epoch \\
\hline 1 & Average Filter & 78.39 & 74.76 & 0.525 & 0.470 & $1 \mathrm{Sec}$ \\
2 & Median Filter & 77.3 & 77.59 & 0.464 & 0.474 & $2 \mathrm{Sec}$ \\
3 & Gaussian Filter & 77.37 & 75.71 & 0.467 & 0.491 & $1 \mathrm{Sec}$ \\
4 & Bilateral Filter & 77.7 & 74.95 & 0.473 & 0.478 & $1 \mathrm{Sec}$ \\
5 & Hybrid Filter & 79.59 & 78.72 & 0.438 & 0.491 & $1 \mathrm{Sec}$ \\
6 & Without Filtering & 65.2 & 69.68 & 0.701 & 0.605 & $1 \mathrm{Sec}$ \\
\hline
\end{tabular}

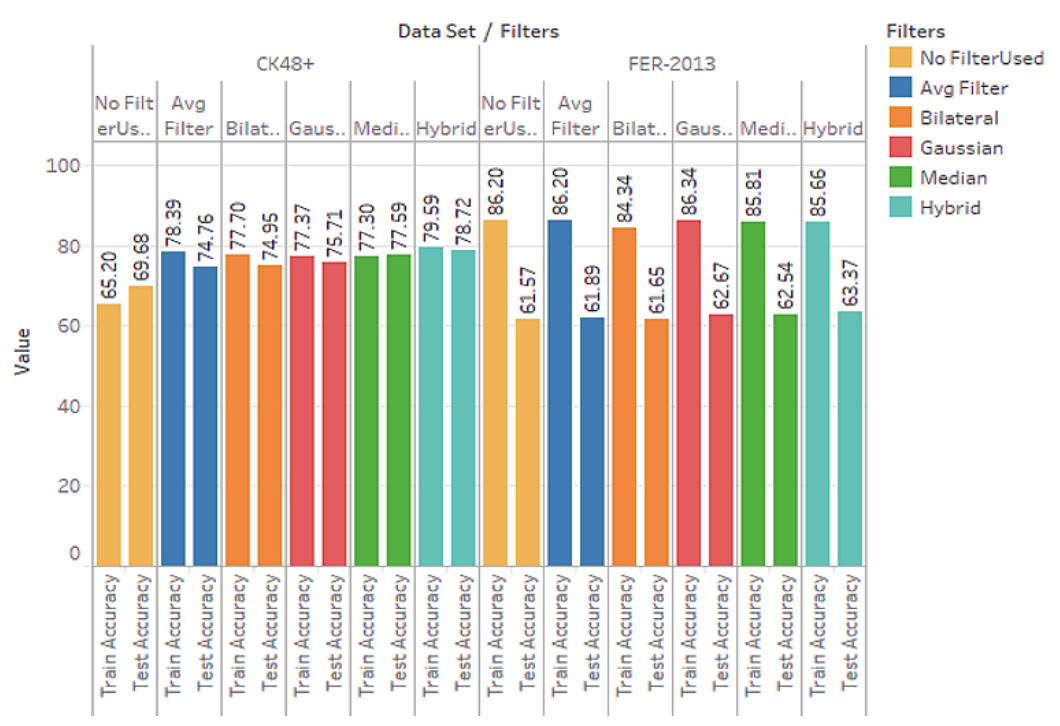

Figure 6. Accuracy comparisons after applying various filters on CK48+ and FER2013 datasets

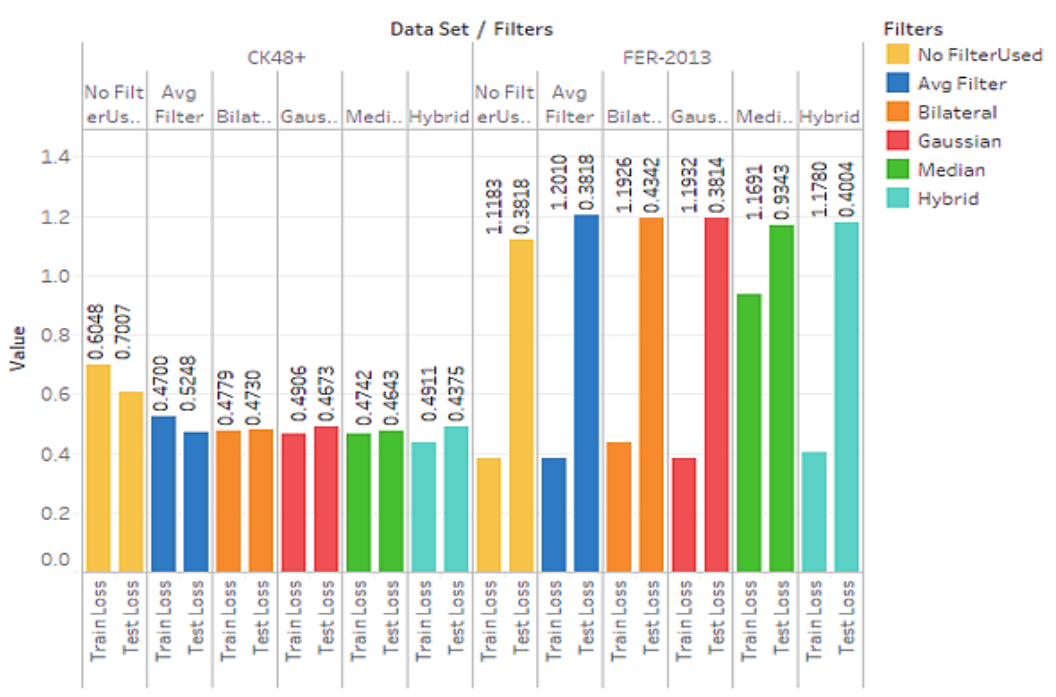

Figure 7. Loss comparisons after applying various filters on CK48+ and FER2013 datasets 


\section{CONCLUSION}

The research described in this article is a robust deep convolutional neural network (CNN) model for facial emotion recognition into one of the seven classes. In this proposed model the input is a mixture of smoothed images produced by different smoothing filters. The model resulted in reasonable performance in terms of accuracy, loss on the test dataset trained using CK48+ and FER 2013 mixed smoothed images. This can be extended to find out most suitable filter for an image which may further increase the accuracy level

\section{REFERENCES}

[1] R. C. Gonzalez and R. E. Woods, "Digital image processing, global edition," $4^{\text {th }}$ Ed., New York: Pearson, pp. 203303, 2018.

[2] E. R. Davies, "Image filtering and morphology," in Computer Vision, $4^{\text {th }}$ Ed., Academic Press, pp. 39-92, 2018, doi: 10.1016/B978-0-12-809284-2.00003-4.

[3] J. Y. W Jien, A. Baharun, S. H. A. Wahab, N. Saad, M. Omar, and N. A. M. Noor, "Age-based facial recognition using convoluted neural network deep learning algorithm," IAES International Journal of Artificial Intelligence (IJAI), vol. 9, no. 3, pp. 424-428, Sep. 2020, doi: 10.11591/ijai.v9.i3.pp424-428.

[4] D. Keltner, "Born to be good: the science of a meaningful life," in Born to Be Good, New York: W.W. Norton and Company, 2009, doi: 10.1080/17439760.2010.516625.

[5] A. Azcarate, F. Hageloh, K. V. D. Sande, and R. Valenti, "Automatic facial emotion recognition," Universiteit van Amsterdam, pp. 1-6, 2005.

[6] Yongmian Zhang and Qiang Ji, "Facial expression understanding in image sequences using dynamic and active visual information fusion," Proceedings Ninth IEEE International Conference on Computer Vision, vol. 2, 2003, pp. 1297-1304, doi: 10.1109/ICCV.2003.1238640.

[7] M. Mohammed, M. V. S. Sownya, Y. Akhila, and B. N. Megana, "Visual modeling of data using convolutional neural networks," International Journal of Engineering and Advanced Technology (IJEAT), vol. 9, no. 1, pp. 49384942, 2019, doi: 10.35940/ijeat.A2084.109119

[8] K Kavitha, B. Sandhya, and B. T. Rao"Evaluation of distance measures for feature based image registration using AlexNet,” arXiv:1907.12921, 2019, doi: 10.14569/IJACSA.2018.091034.

[9] P. Mishra, Raju, and Pevithra, "Crowd counting using CSR-Net architecture," International Journal of Advanced Trends in Computer Science and Engineering, vol 8, no. 6, pp. 2762-2767, 2019, doi: 10.30534/ijatcse/2019/13862019.

[10] P. V. V. Kishore et al., "Indian classical dance action identification and classification with convolutional neural networks," Advances in Multimedia, vol. 2018, 2018, Art. no. 5141402, doi: 10.1155/2018/5141402.

[11] R. B. Roslan, I. N. M. Razly, N. Sabri, and Z. Ibrahim, "Evaluation of psoriasis skin disease classification using convolutional neural network," IAES International Journal of Artificial Intelligence (IJ-AI), vol, 9, no. 2, pp. 349, 2020, doi: 10.11591/ijai.v9.i2.pp349-355.

[12] H. A Abdulkarim, M. A. A. Razak, R. Sudirman, and N. Ramli, "A deep learning AlexNet model for classification of red blood cells in sickle cell anemia," IAES International Journal of Artificial Intelligence (IJ-AI), vol. 9, no. 2, pp. 221-228., 2020, doi: 10.11591/ijai.v9.i2.pp221-228.

[13] S. Z. M. Zaki, M. A. Zulkifley, M. M. Stofa, N. A. M. Kamari, N. A. Mohamed, "Classification of tomato leaf diseases using MobileNet v2," IAES International Journal of Artificial Intelligence (IJ-AI), vol. 9, no. 2, pp. 290296, 2020, doi: 10.11591/ijai.v9.i2.pp290-296.

[14] K. Bhagavan, S. S. Imambi, and S. Bano, "A compressive survey on different image processing techniques to identify the brain tumor," International Journal of Engineering and Technology, vol. 7, no. 2, pp. 1081-1084, 2018, doi: 10.14419/IJET.V7I2.7.12232.

[15] G. Pradeepini, B. S. Babu, T. Tejaswin, D. Priyanka, and M. Harshitha, "A comparative study on brain tumor diagnosis techniques using MRI image processing," International Journal of Engineering and Technology, vol. 7, pp. 486-489, 2018, doi: 10.14419/ijet.v7i2.32.16274.

[16] E. Vamsidhar, P. J. Rani, and K. R. Babu, "Plant disease identification and classification using image processing," International Journal of Engineering and Advanced Technology, vol. 8, pp. 442-446, 2019.

[17] S. K. L. Praneetha, K. Anusha, R. G. Viharika, M. D. Sree, and P. Vidyullatha, "Automated leaf disease detection in corn species through image analysis," International Journal of Advanced Trends in Computer Science and Engineering, vol. 8, no. 6, pp. 2893-2899, 2019, doi: 10.30534/ijatcse/2019/34862019

[18] P. R. Keerthi, B. Niharika, G. D. Kumar, K. S. Venakat, and C. M. S. Rani, "Reorganization of license plate characteristics using image processing techniques," International Journal of Recent Technology and Engineering, vol. 7, no. 6, pp. 1260-1264, 2019.

[19] S. Inthiyaz, B. T. P. Madhav, and P. V. V. Kishore, "Flower image segmentation with PCA fused colored covariance and gabor texture features based level sets," Ain Shams Engineering Journal, vol. 9, no. 4, pp. 3277 3291, 2018, doi: 10.1016/j.asej.2017.12.007.

[20] P. V. V. S. Srinivas and P. Mishra, "Facial Expression Detection Model of Seven Expression Types Using Hybrid Feature Selection and Deep CNN," in International Conference on Intelligent and Smart Computing in Data Analytics: ISCDA 2020, 2021, pp. 89-101, vol. 1312, doi: 10.1007/978-981-33-6176-8_10.

[21] C.-T. Lu and T.-C. Chou, "Denoising of salt-and-pepper noise corrupted image using modified directionalweighted-median filter," Pattern Recognition Letters, vol. 33, no. 10, pp. 1287-1295, 2012, doi: 10.1016/j.patrec.2012.03.025. 
[22] A. Shah et al., "Comparative analysis of median filter and its variants for removal of impulse noise from gray scale images," Journal of King Saud University - Computer and Information Sciences, 2020, doi: 10.1016/j.jksuci.2020.03.007.

[23] V. Gregori, S. Morillas, B. Roig, A. Sapena, "Fuzzy averaging filter for impulse noise reduction in colour images with a correction step," Journal of Visual Communication and Image Representation, pp. 518-528, 2018, doi: 10.1016/j.jvcir.2018.06.025.

[24] A. F. de Araujo, C. E. Constantinou, and J. M. R. S. Taves, "Smoothing of ultrasound images using a new selective average filter," Expert Systems with Applications: An International Journal, pp. 96-106, 2016, doi: 10.1016/j.eswa.2016.04.034.

[25] Y. Zhu and C. Huang, "An improved median filtering algorithm for image noise reduction," Physics Procedia, vol. 25, pp. 609-616, 2012, doi: 10.1016/j.phpro.2012.03.133.

[26] M. H. Hsieh, F. C. Cheng, M. C. Shie, and S. J, Ruan, "Fast and efficient median filter for removing 1-99\% levels of salt-and-pepper noise in images," Engineering Applications of Artificial Intelligence, vol. 26, no. 4, pp. 13331338, 2013, doi: 10.1016/j.engappai.2012.10.012.

[27] C. Zhang and K. Wang, J. Li, and S. K. Jha, "A switching median-mean filter for removal of high-density impulse noise from digital images," Optik, vol. 126, no. 9-10, pp. 956-961, 2015, doi: 10.1016/j.ijleo.2015.02.085.

[28] L. Shapiro and G. Stockman, "Computer Vision," Pearson, pp. 137-150, 2001.

[29] J. Wang, G. Wu, J. Li, and S. K. Jha "A new method estimating linear Gaussian filter kernel by image PRNU noise," Journal of Information Security and Applications, vol. 44, pp. 1-11, 2019, doi: 10.1016/j.jisa.2018.11.002.

[30] H. Yin, Y. Gong, and G. Qiu, "Fast and efficient implementation of image filtering using a side window convolutional neural network," Signal Processing, vol. 176, 2020, Art. No. 107717, doi: 10.1016/j.sigpro.2020.107717.

[31] C. Tomasi and R. Manduchi, "Bilateral filtering for gray and color images," Sixth International Conference on Computer Vision (IEEE Cat. No.98CH36271), 1998, pp. 839-846, doi: 10.1109/ICCV.1998.710815.

[32] S. Routray, P. P. Malla, S. K. Sharma, S. K. Panda, and G. Palai, "A new image denoising framework using bilateral filtering based non-subsampled shearlet transform," Optik, 2020, Art. No. 164903, doi: 10.1016/j.ijleo.2020.164903.

[33] L. Jin, C. Xiong, and H. Liu, "Improved bilateral filter for suppressing mixed noise in color images," Digital Signal Processing, vol. 22, no. 6, pp. 903-912, 2012, doi: 10.1016/j.dsp.2012.06.012.

\section{BIOGRAPHIES OF AUTHORS}

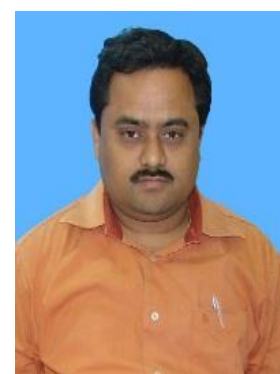

Mr. Pragnyaban Mishra is working on the Koneru Lakshmaiah Education Foundation, Andhra Pradesh, India as an Associate Professor since the year 2017 which is one of the reputed University of India having NIRF rank 41 ranked by Govt. Of India. He has published around 25 research publications out of which eleven are indexed in SCOPUS and two are in Web of Science database. One PhD scholar awarded, two are yet to be awarded and five are actively doing research under the direct supervision of Mr. Mishra. The subjects he had taught to UG and PG students are listed as Data Visualization, Computer Vision, Deep Learning, Machine Learning and Data Science. Besides research and teaching, he served the university as a member of the different academic committee to his capacity and level best.

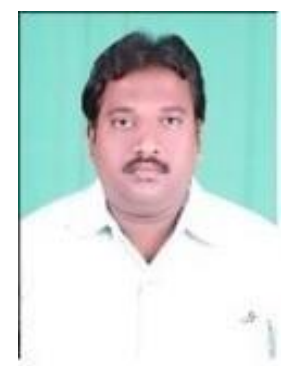

Mr. PVVS Srinivas is a Research Scholar of the Department of CSE, Koneru Lakshmaiah Education Foundation, Andhra Pradesh, India. He is actively involved in developing models using Artificial Intelligence. He has published three research papers that are indexed in SCOPUS database. Mr. Srinivas taught the subjects like Data Visualization, Computer Vision, Deep Learning, Machine Learning and Data Science to the UG students of the university where he is pursuing $a \mathrm{PhD}$. 